\title{
Spectroscopic Study of the Interaction of DNA with the Linker Histone H1 from Starfish Sperm Reveals Mechanisms of the Formation of Supercondensed Sperm Chromatin
}

\author{
Elena Chikhirzhina, ${ }^{1}$ Tatyana Starkova, ${ }^{1,2}$ Elena Kostyleva, ${ }^{1}$ and Alexander Polyanichko ${ }^{1,2}$ \\ ${ }^{1}$ Laboratory of Cell Biochemistry, Institute of Cytology, Russian Academy of Sciences, \\ Saint-Petersburg 194064, Russia \\ ${ }^{2}$ Deprtment of Molecular Biophysics, Faculty of Physics, Saint-Petersburg State University, \\ Saint-Petersburg 198504, Russia \\ Correspondence should be addressed to Elena Chikhirzhina, chikhir@gmail.com \\ Copyright (C) 2012 Elena Chikhirzhina et al. This is an open access article distributed under the Creative Commons Attribution \\ License, which permits unrestricted use, distribution, and reproduction in any medium, provided the original work is properly \\ cited.
}

\begin{abstract}
The interaction of the linker histone H1Z from the sperm chromatin of starfish Asterias amurensis with DNA was studied by spectroscopic and thermodynamic approaches. It has been shown that at the physiological conditions the interaction of the H1Z with DNA results in more compact structures compared to complexes of DNA with somatic histone H1. The typical profile of the DNA melting curves reveals two peaks attributed to the bound and unbound DNA. It has been shown that H1Z from starfish sperm stabilizes DNA to a greater extent compared to the somatic H1. It is possible that the presence of the additional $\alpha$-helical segments within the C-terminal part of the H1Z typical for the linker histones from echinoderm sperm facilitates the protein-protein interactions which in turn stimulate cooperative binding of the histones to DNA, resulting in the formation of the supercompact sperm chromatin.
\end{abstract}

Keywords: Chromatin, circular cichroism, DNA, DNA melting, histone H1

\section{Introduction}

Chromatin of eukaryotic cells comprises DNA and variety of nuclear proteins. One of the important functions of the chromatin is the DNA compaction, whose linear dimensions are much larger than the diameter of a nucleus [1]. The proteins responsible for the initial level of the DNA compaction, also known as the nucleosomal level, are histones. There are 5 different types of histones in the chromatin: H1, H2A, H2B, H3, and H4 [1, 2]. Four core histones interact with each other and with DNA forming a nucleosome. The targets for the histone $\mathrm{H} 1$ are the linker regions of DNA between the nucleosomes. 
Linker histone $\mathrm{H} 1$ plays an essential role in the post nucleosomal level of the structural organization of the chromatin [1]. Different cells demonstrate different level of DNA compaction, and one of the most compact chromatin can be found in sperm cells. In these cells, histone H1 is replaced by other different proteins, such as sperm-specific histones, thioprotamines, or protamines [1, 3]. In those cells, where linker histone $\mathrm{H} 1$ is substituted by the sperm-specific proteins, the chromatin retains the nucleosomal organization, still being considerably more compact then the chromatin of somatic cells. It makes the sperm-specific proteins a convenient model object to study the mechanisms of DNA compaction in chromatin.

In this study, we investigated the interaction of the H1 extracted from sperm of starfish Asterias amurensis with DNA using circular dichroism (CD) spectroscopy and thermal melting of DNA.

\section{Materials and Methods}

Linker histones $\mathrm{H} 1$ were isolated from rat thymus and from chromatin of starfish Astorias amurensis (H1-Z) sperm as described earlier [4]. Chicken erythrocyte DNA was characterized as described earlier [5] and used without further purification. To prepare artificial DNA-protein complexes low-molecularweight DNA with a mean size of $550 \mathrm{bp}$ was used, obtained by sonication at $22 \mathrm{kHz}$ as described earlier [6].

The protein content in the system was described in terms of the molar ratio of the protein amino acid residues to DNA phosphates $(r)$, which varied from 0 to 2 . All the complexes were studied in buffer solution containing $5 \mathrm{mM}$ Tris- $\mathrm{HCl}, \mathrm{pH} 7.5,1 \mathrm{mM}$ EDTA in the presence of $15 \mathrm{mM} \mathrm{NaCl}$ (low ionic strength) or $150 \mathrm{mM} \mathrm{NaCl}$ (high ionic strength).

Spectra of the circular dichroism (CD) were recorded in terms of ellipticity $\theta$ using Cary $60 \mathrm{CD}$ spectropolarimeter-dichrograph (Varian, USA) in round quartz cells with the optical path length of $1 \mathrm{~cm}$. Precision of the measurements amounted to $\pm 0.0002^{\circ}$. The spectra were recorded in the range of 195 to $320 \mathrm{~nm}$. Values of molar ellipticity $[\theta](\operatorname{grad~cm} 2 / \mathrm{dmol})$ were calculated per mole of DNA nucleotides $\left(M_{0}=330\right)$. The instrument was calibrated with solution of D-10-camphorosulfonic acid in the spectral range of $190-290 \mathrm{~nm}$.

The thermal stability of DNA in the complexes with histones was studied using the DNA melting analysis. The melting curves were recorded spectrophotometrically using Specord M40 double-beam scanning spectrophotometer (Carl Zeiss, Jena, Germany) supplied with the TSEI Peltier attachment. The error of the transmission measurements was equal to $\pm 0.01 \% T$. The optical densities $\mathrm{D}$ of the solutions were measured in rectangular quartz cells with the optical path length of $1 \mathrm{~cm}$. The rate of heating was maintained at $0.5^{\circ} \mathrm{C}$ per minute. The melting curves $D_{260}(T)$ were smoothed using Savitzky-Golay treatment. The normalized melting curves $h(T)$ were obtained using the following relationship:

$$
h(T)=\frac{D_{260}(T)-D_{260}^{\min }}{D_{260}^{\max }-D_{260}^{\min }}
$$

where $D_{260}^{\min }$ and $D_{260}^{\max }$ are minimum and maximum values of the optical density at $260 \mathrm{~nm}$ corresponding to the native and melted DNA states in the process of melting. 
Derivative melting curves $d h(T) / d T$ were deconvoluted to Gaussian bands and contribution of each band was determined. The DNA-protein complexes were characterized by the value of hyperchromic effect $G$, by melting temperature $T_{m}$, and by the fraction of DNA melted.

The hyperchromic effect $G$ was determined by the formula:

$$
G=\frac{D_{260}^{\max }-D_{260}^{\min }}{D_{260}^{\min }} \times 100 \%
$$

The melting temperature $T_{m}$ was determined as the maximum of the derivative melting curve $d h(T) / d T$.

\section{Results and Discussion}

Compared to the somatic H1 the sperm-specific histones are characterized by elongated arginine-rich polypeptide chain and by presence of basic amino acid sequences (Ser-Pro-Lys(Arg)-Lys(Arg)) [1]. These peculiarities can be responsible for their structural properties and underlie the mechanisms of the supercondensation of DNA in the sperm chromatin. Although the proteins are similar in terms of their secondary structure, the proteins from echinoderm sperm demonstrate higher content of $\alpha$ helical regions in their C-terminal domains compared to the somatic H1 [4]. Such regions of the polypeptide chain, as well as the globular $\alpha$-helical regions, are rather unstable and melt at relatively low temperatures.

To follow the changes in DNA structure during the interaction with the starfish sperm histones, we used circular dichroism spectroscopy. Figures 1 and 2 demonstrate CD spectra of the complexes between DNA and $\mathrm{H} 1$ from the rat thymus and the starfish sperm in the solutions of low and high ionic strength, respectively.

Increasing concentration of the H1 from rat thymus in the complex $(r<2.0)$ does not lead to significant changes in the intensity of positive CD band in the range 270-280 nm (Figure 1(a)) in the solutions of $15 \mathrm{mM} \mathrm{NaCl}$ revealing no considerable changes in the DNA structure. Increasing influence of the light scattering on the shape of spectra of the complexes between DNA and histone H1 from the starfish at $1.5<r<2.0$ reveals intensive intermolecular interactions in the solution (Figure 1(b)).

Increasing intensity of the negative $C D$ band in the range of $210-230 \mathrm{~nm}$ shows that the interaction of histone $\mathrm{H} 1$ with the DNA leads to the partial spiralization of the protein.

In solutions of high ionic strength $(150 \mathrm{mM} \mathrm{NaCl})$, the interaction of DNA with the protein molecules results in DNA condensation, which manifests itself as so-called $\Psi$-type CD spectrum of DNA (PSI: Polymer and Salt Induced) [7]. This CD spectrum is characterized by deep negative or positive band in the range of $240-300 \mathrm{~nm}$ and long-wavelength "tail" in CD spectra. Figure 2 represents CD spectra of DNA-H1 complexes at high ionic strength solution. The beginning of the condensation of the DNA in the complex with the somatic histone H1 (Figure 2(a)) at $r=1.5$ manifests itself in a notable light scattering resulting in the shift of the positive CD band of DNA toward the longer wavelengths and the appearance of the spectral "tail" in the region above $290 \mathrm{~nm}$. At $r=2.0$, the CD spectrum of the DNA-H1T transforms to a typical $\Psi$-type CD spectrum. In the case of H1 from starfish sperm (Figure 2(b)), even a small amount of the protein leads to increasing amplitude of the positive CD band of DNA accompanied by a considerable light scattering in the solution. Formation of the very typical 


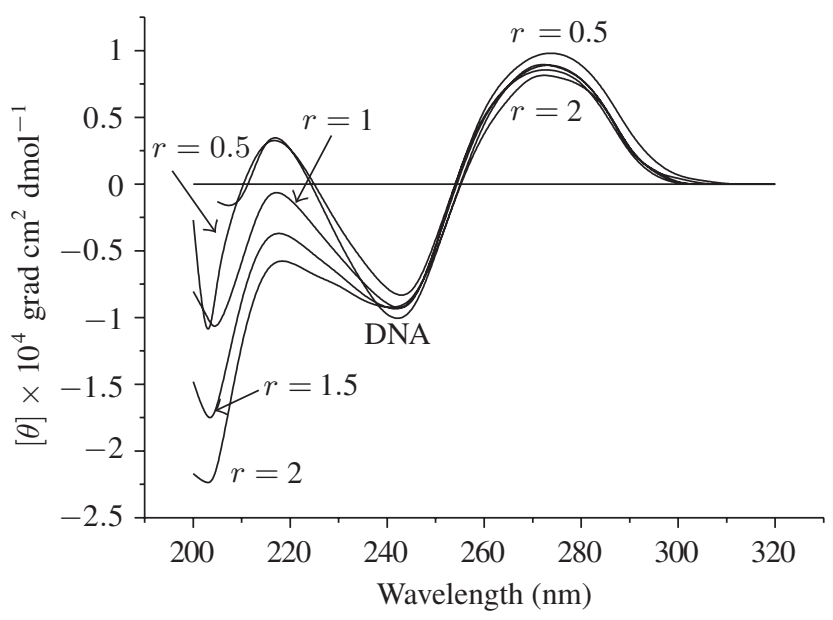

(a)

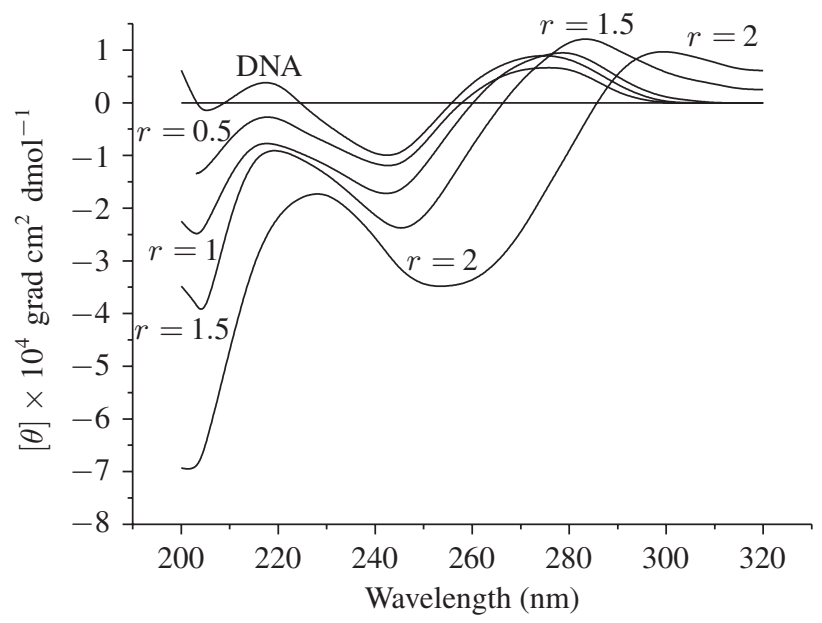

(b)

Figure 1: CD spectra of the complexes of DNA with the linker histone H1T from rat thymus (a) and $\mathrm{H} 1 \mathrm{Z}$ from starfish sperm (b) in $15 \mathrm{mM} \mathrm{NaCl}$ at different protein to DNA ratios $(r)$.

$\Psi$-type CD spectrum indicates the formation of the ordered supramolecular DNA-protein structures in the solution [5, 8-10]. Most likely, the prerequisite for the formation of such ordered structures is the interaction between the hydrophobic clusters of the histone molecules. Indeed, such clusters occur in the $\mathrm{H} 1$ from echinoderm sperm more frequently than in the somatic proteins [4] and these histones do compact DNA to a greater extent. It should be noted that the additional $\alpha$-helical regions of histone $\mathrm{H} 1$ from the spermatozoa of echinoderms have been found in the C-terminal domain of the protein. In particular, this domain is rich in alanine and lysine amino acid residues [11], and it is responsible for the ability of the protein to compact DNA [12].

To study the ability of the proteins to stabilize the DNA structure when complexed with DNA we obtained melting curves of the pure DNA and the DNA in the complex with sperm-specific histone 


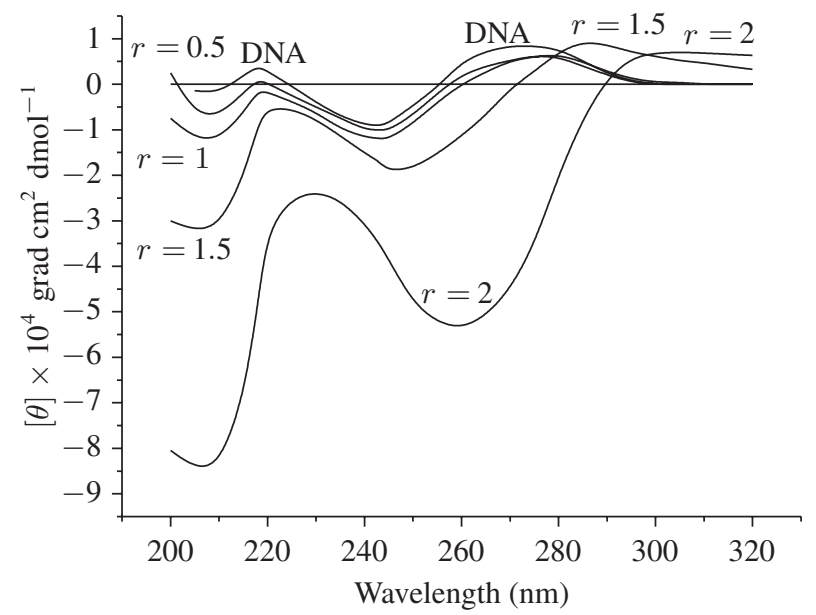

(a)

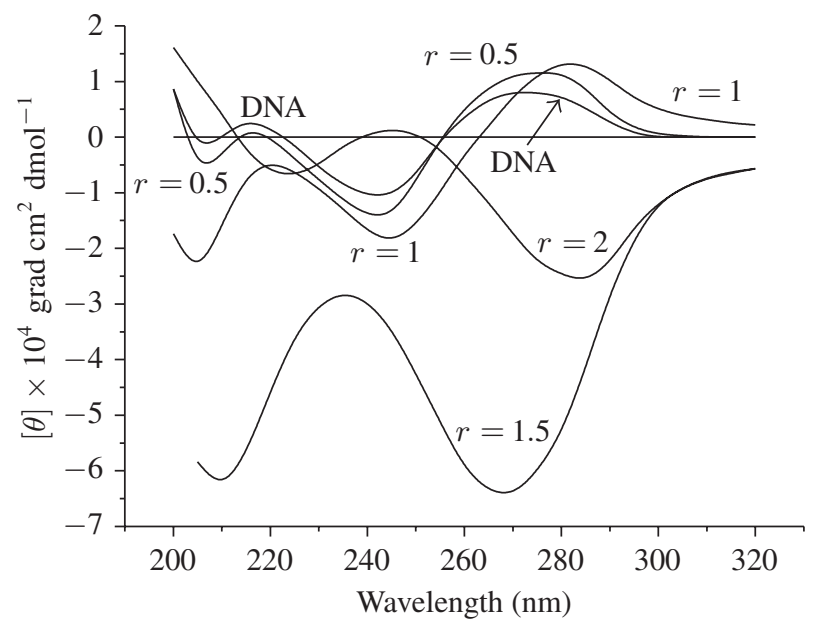

(b)

Figure 2: CD spectra of the complexes of DNA with the linker histone H1T from rat thymus (a) and $\mathrm{H} 1 \mathrm{Z}$ from starfish sperm (b) in $150 \mathrm{mM} \mathrm{NaCl}$ at different protein to DNA ratios $(r)$.

H1 from starfish sperm chromatin. The melting temperature of DNA depends on the ionic strength and ionic composition of solution. To reduce the influence of the possible traces of divalent ions, we added to the solutions $0.25 \mathrm{mM}$ EDTA.

The typical derivative melting curves of the DNA-protein complexes have two peaks (Figure 3): the low-temperature peak $T_{m I}$ corresponds to the melting of free DNA and the higher temperature peak $T_{m I I}$ characterizes the melting of DNA bound to $\mathrm{H} 1$ protein [13]. The melting temperature of DNA in the complex depends on the particular histone, whereas the melting temperature of the unbound DNA remains constant for all complexes. The presence of two peaks shows that even at high protein to DNA ratio $(r=1.0)$, there is the unbound fraction of DNA in the solution. Figure 3 shows that the melting temperatures of the bound DNA fractions in all complexes have higher melting temperatures, 


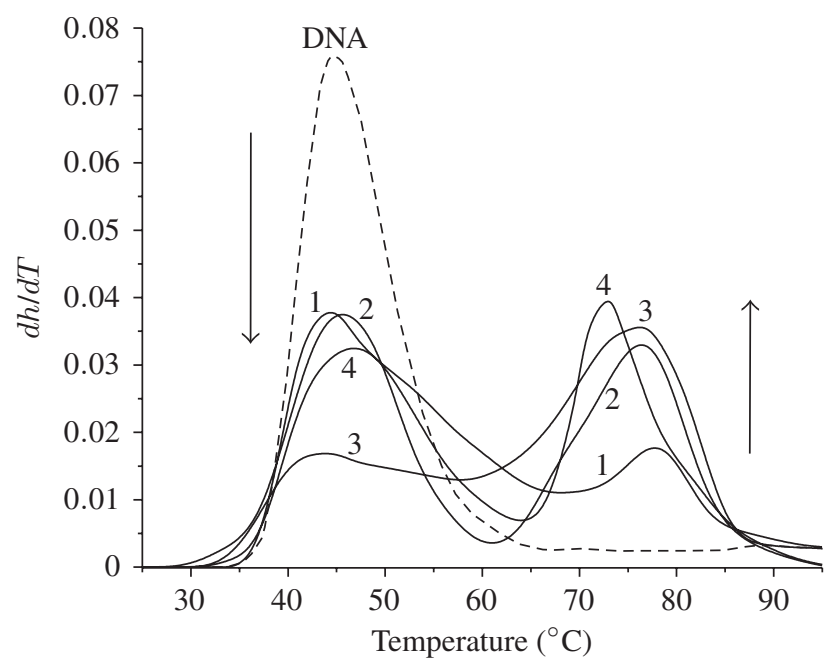

Figure 3: Derivative melting curves of the complexes of DNA with the linker histone H1 from starfish sperm in $0.25 \mathrm{mM}$ EDTA at different protein to DNA ratios $(r)$ : DNA (dash); (1) DNA-H1Z, $r=0.5$; (2) DNA-H1Z, $r=1.0$; (3) DNA-H1Z, $r=1.5$; (4) DNA-H1T, $r=1.0$.

which do not depend on $r$ (Table 1). The area under each peak is proportional to the amount of DNA in the corresponding fraction: unbound DNA for $T_{m I}$ and bound DNA for $T_{m I I}$. The gradual changes in the areas of the both peaks characterize the increasing number of DNA segments bound to protein with increasing protein concentration. The total area under the derivative curve is constant at all DNA to protein ratios in solution. The exact number of the bound/unbound DNA segments depends not only on the amount of protein but also on the type of histone (Table 1). Figure 3 also represents the melting curve for DNA-H1T (somatic histone H1) complex at $r=1.0$. The comparison of the two melting curves for DNA-H1Z and DNA-H1T (Figure 3, Table 1) shows that, at the equimolar DNA to protein ratio, the fraction of the unbound DNA is larger in the DNA-H1Z complexes.

The analysis of the DNA melting curves also demonstrates that the most basic (positively charged) H1 from starfish sperm stabilizes DNA to a greater extent while the DNA conformation in the complexes with the less charged somatic H1 from rat thymus is also less stable (Figure 3, Table 1). Such differences might be due to the fact that the sperm-specific histones interact with DNA not only in the major groove but also in the minor groove [5]. In this case, intramolecular crosslinks on the DNA are created and the sites enriched in protein appear on DNA. However, in the case of the interaction of DNA with starfish sperm H1 such are fewer than for DNA-H1T complexes. This can be explained by an increased content of arginine in the sperm-specific proteins, which stabilize DNA screening the negative charges of the phosphate groups [13].

The differences in the DNA binding properties and in the ability of the complexes to aggregate are likely depended on the conformational properties of the histones. The cooperative binding to DNA and stronger condensation potential are the characteristic properties of the echinoderm sperm H1 histones. These histones have approximately the same content of lysine, but more alanine, and less proline residues than the somatic protein [14]. The polypeptide chain of $\mathrm{H} 1$ histone forms a random coil at low ionic strength and at acidic $\mathrm{pH}$. It is possible that the presence of additional $\alpha$-helical segments 
Table 1: The main thermodynamic characteristics of the temperature-induced conformational transition of free DNA and of the DNA in the complexes with H1 starfish sperm (DNA-H1Z) and somatic H1 (DNA-H1T) in $0.25 \mathrm{mM}$ EDTA at the different ratios of protein amino acid residues to DNA phosphates.

\begin{tabular}{lccccc}
\hline & DNA & $\begin{array}{c}\text { DNA-HIZ } \\
r=0.5\end{array}$ & $\begin{array}{c}\text { DNA-HIZ } \\
r=1.0\end{array}$ & $\begin{array}{c}\text { DNA-HIZ } \\
r=1.5\end{array}$ & $\begin{array}{c}\text { DNA-HIT } \\
r=1.0\end{array}$ \\
\hline $\begin{array}{l}\text { Hyperchromiceffect } \\
G, \%\end{array}$ & $35.1 \pm 0.5$ & $39.8 \pm 0.5$ & $39.2 \pm 0.5$ & $35.7 \pm 0.5$ & $39.3 \pm 0.5$ \\
$T_{m I},{ }^{\circ} \mathrm{C}$ & $45.0 \pm 0.5$ & $45.0 \pm 0.5$ & $45.0 \pm 0.5$ & $45.0 \pm 0.5$ & $45.0 \pm 0.5$ \\
$T_{m I I},{ }^{\circ} \mathrm{C}$ & - & $77.0 \pm 0.5$ & $77.0 \pm 0.5$ & $77.0 \pm 0.5$ & $73.0 \pm 0.5$ \\
$\begin{array}{l}\text { Fraction of DNA } \\
\text { Melted at } T_{m I}, \%\end{array}$ & $100 \pm 0.5$ & $71.0 \pm 0.5$ & $54.0 \pm 0.5$ & $37.0 \pm 0.5$ & $51.0 \pm 0.5$ \\
$\begin{array}{l}\text { Fraction of DNA } \\
\text { Melted at } T_{m I I}, \%\end{array}$ & 0 & $29.0 \pm 0.5$ & $46.0 \pm 0.5$ & $63.0 \pm 0.5$ & $49.0 \pm 0.5$ \\
\hline
\end{tabular}

which we found in the C-terminal part of the $\mathrm{H} 1$ from echinoderm sperm $[4,5]$ facilitates the proteinprotein interactions in solution $[10,15]$. The latter in turn stimulate cooperative binding of the histones of this type to DNA, resulting in the formation of ordered structures in the chromatin.

\section{Acknowledgments}

The authors are grateful to the Russian Foundation for Basic Researches for a financial support (10-0400092), the Government of St. Petersburg, Federal Program "Scientific and pedagogical labor force for an innovative Russia (P1163).”

\section{References}

[1] E. V. Chikhirzhina and V. I. Vorob'ev, "Linker histones: conformational changes and the role in the structural organization of chromatin," Tsitologiya, vol. 44, no. 8, pp. 721-736, 2002.

[2] R. D. Kornberg, "Chromatin structure: a repeating unit of histones and DNA," Science, vol. 184, no. 4139, pp. 868-871, 1974.

[3] J. M. Eirin-Lopez and J. Ausio, "Origin and evolution of chromosomal sperm proteins," BioEssays, vol. 31, no. 10, pp. 1062-1070, 2009.

[4] E. I. Ramm, E. V. Chikhirzhina, E. N. Kostyleva, and V. I. Vorob'ev, "Conformational features of linker proteins of supercompact chromatin from marine invertebrate sperm," Biokhimiya, vol. 60, no. 1 , pp. 150-158, 1995.

[5] E. V. Chikhirzhina, E. I. Kostyleva, E. I. Ramm, and V. I. Vorob'ev, "Chromatin compactification using a model system of DNA-protein complexes," Tsitologiia, vol. 40, no. 10, pp. 883-888, 1998.

[6] E. V. Chikhirzhina, T. Y. Starkova, E. I. Kostyleva, G. I. Chikhirzhina, V. I. Vorobiev, and A. M. Polyanichko, "Interaction of DNA with sperm-specific histones of the H1 family," Cell and Tissue Biology, vol. 5, no. 6, pp. 536-542, 2011.

[7] C. F. Jordan, L. S. Lerman, and J. H. Venable, "Structure and circular dichroism of DNA in concentrated polymer solutions.," Nature: New biology, vol. 236, no. 64, pp. 67-70, 1972. 
[8] E. V. Chikhirzhina, A. M. Polyanichko, A. N. Skvortsov, E. I. Kostyleva, C. Houssier, and V. I. Vorob'ev, "HMG1 domains: The victims of the circumstances," Molecular Biology, vol. 36, no. 3, pp. 412-418, 2002.

[9] A. M. Polyanichko, E. V. Chikhirzhina, A. N. Skvortsov et al., "The HMG1 Ta(i)le," Journal of Biomolecular Structure and Dynamics, vol. 19, no. 6, pp. 1053-1062, 2002.

[10] A. M. Polyanichko, Z. V. Leonenko, D. Cramb, H. Wieser, V. I. Vorob'ev, and E. V. Chikhirzhina, "Visualization of DNA complexes with HMGB1 and its C-truncated form HMGB1(A+B)," Biophysics, vol. 53, no. 3, pp. 202-206, 2008.

[11] C. S. Hill, S. R. Martin, and J. O. Thomas, "A stable $\alpha$-helical element in the carboxy-terminal domain of free and chromatin-bound histone H1 from sea urchin sperm," EMBO Journal, vol. 8, no. 9, pp. 2591-2599, 1989.

[12] J. Allan, T. Mitchell, and N. Harborne, "Roles of H1 domains in determining higher order chromatin structure and H1 location," Journal of Molecular Biology, vol. 187, no. 4, pp. 591-601, 1986.

[13] E. I. Ramm, G. S. Ivanov, V. I. Vorob'ev, S. N. Kadura, and S. N. Khrapunov, "Structure of illexine I2 and its complexes with DNA," Molekulyarnaya Biologiya, vol. 21, no. 6, pp. 1590-1599, 1987.

[14] I. A. Zalenskaya, E. O. Zalenskaya, and A. O. Zalensky, "Basic chromosomal proteins of marine invertebrates-II. Starfish and holothuria," Comparative Biochemistry and Physiology. Part B, vol. 65B, no. 2, pp. 375-378, 1980.

[15] E. Chikhirzhina, A. Polyanichko, Z. Leonenko, H. Wieser, and V. Vorob'ev, "C-terminal domain of nonhistone protein HMGB1 as a modulator of HMGB1-DNA structural interactions," Spectroscopy, vol. 24, no. 3-4, pp. 361-366, 2010. 


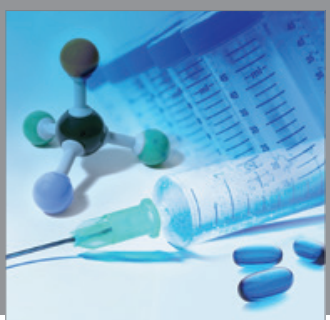

International Journal of

Medicinal Chemistry

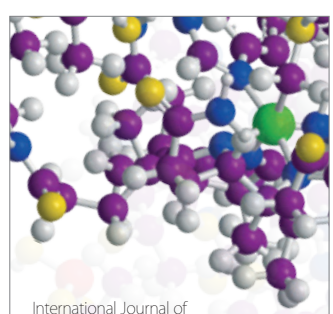

Carbohydrate Chemistry

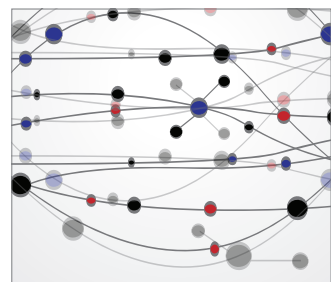

The Scientific World Journal
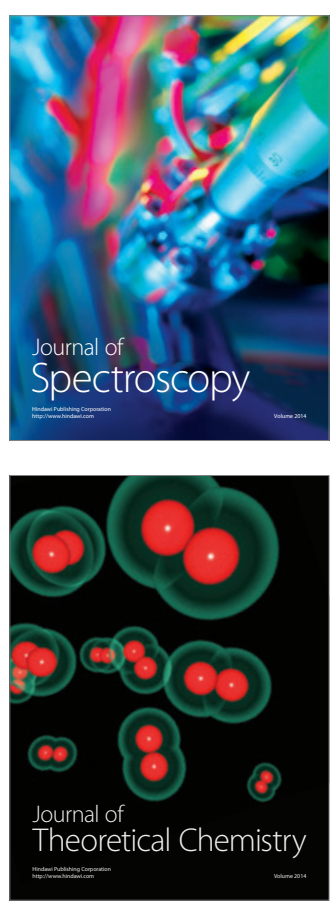
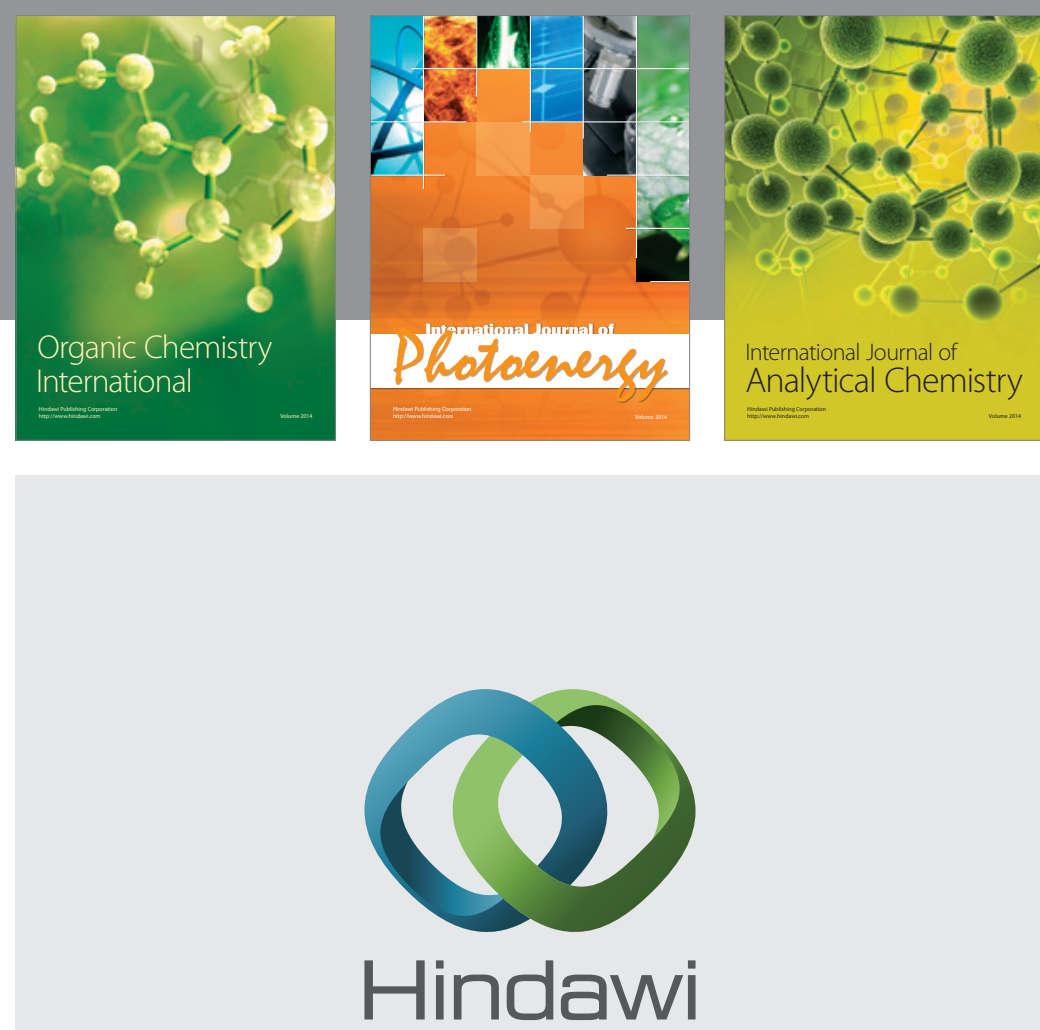

Submit your manuscripts at

http://www.hindawi.com
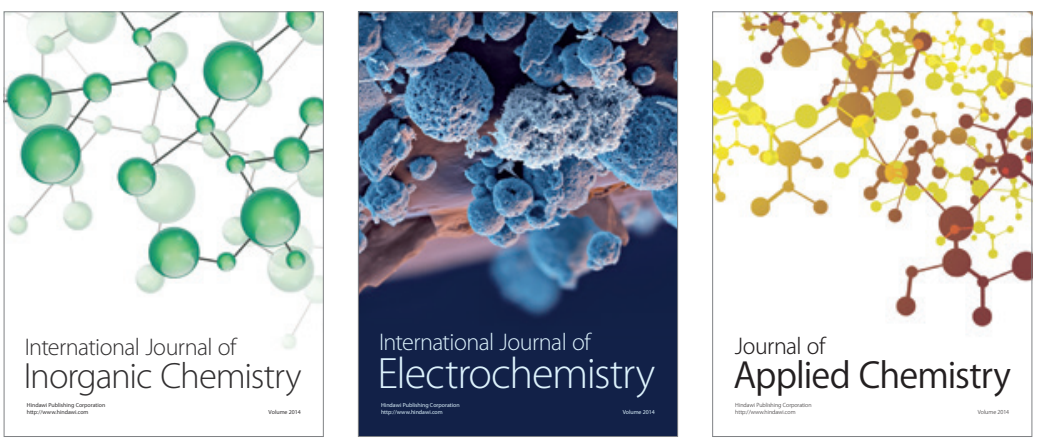

Journal of

Applied Chemistry
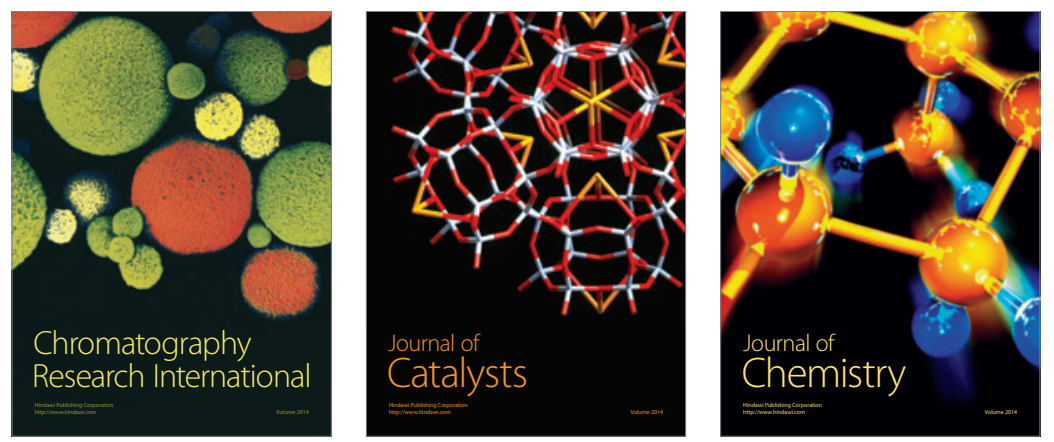
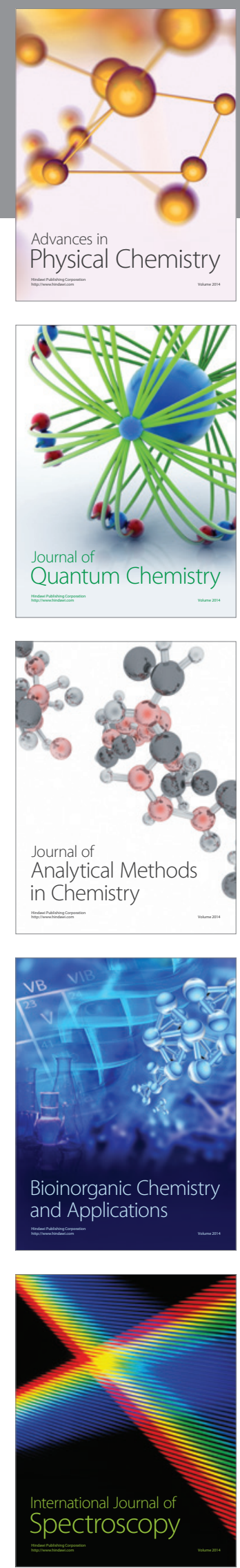